\title{
Nuevas fórmulas publicitarias: los advergames como herramienta de las comunicaciones de marketing
}

\author{
New advertising formats: advergames as a \\ marketing communication tool
}

\author{
José Martí Parreño \\ Rafael Currás Pérez ${ }^{1}$ \\ IsABel SÁnchez García ${ }^{1}$ \\ Universitat de València (España)
}

Recibido el 17 de mayo de 2010 y aceptado el 24 de noviembre de 2010

$\mathrm{N}^{\mathrm{o}}$ de clasificación JEL: M37

DOI: $10.5295 / \mathrm{cdg} .100236 \mathrm{jm}$

\section{Resumen:}

Los advergames son videojuegos orientados a la comunicación publicitaria. Su naturaleza de mensaje híbrido, al combinar contenido publicitario y contenido de entretenimiento, aporta interesantes posibilidades al desarrollo de comunicaciones de marketing en un entorno mediático en el que la publicidad tradicional ha ido perdiendo eficacia. El presente artículo delimita el concepto de advergame, analizando las diferentes tipologías existentes y sus efectos sobre diversos objetivos de marketing y de comunicación. Asimismo, se apuntan algunas recomendaciones a la hora de desarrollar comunicaciones de marketing mediante advergames, así como las cuestiones éticas y morales que deben considerarse.

Palabras clave:

Publicidad, medios alternativos, videojuegos, advergames.

\begin{abstract}
:
Advergames are marketing communication-driven videogames. As a hybrid message, they combine advertising content and entertainment content, providing interesting opportunities to marketing communications in a new media environment where traditional advertising has lost effectiveness. This paper clarifies the concept of advergame, analysing the different types of advergames and their effects on marketing and communication goals. Furthermore some recommendations for implementing marketing communication through advergames are provided, as well as ethic and moral issues that should be considered.
\end{abstract}

Keywords:

Advertising, alternative media, videogames, advergames.

\footnotetext{
${ }^{1}$ Universitat de València, Facultad de Economía, Dpto. Comercialización e Investigación de Mercados, Avda. Tarongers s/n 46022 Valencia, España.Email: jose.marti-parreno@uv.es rafael.curras-perez@uv.es isabel.sanchez@ uv.es
} 


\section{INTRODUCCIÓN}

Hoy en día las organizaciones tienen que adaptarse a un nuevo contexto de mercado caracterizado por una demanda creciente de experiencias únicas y personalizadas que llevan a las empresas a buscar nuevas formas de aportar valor añadido a clientes y consumidores potenciales. En este contexto, las comunicaciones de masas que predominaban en el pasado ya no son efectivas, siendo reemplazadas cada vez más por comunicaciones bidireccionales en medios interactivos que sean capaces de aportar nuevos valores (como el entretenimiento) a los consumidores.

Por otra parte, el creciente número de medios y soportes en los que desarrollar estas comunicaciones de marketing, la saturación publicitaria de los mismos y la fragmentación de audiencias de este nuevo ecosistema mediático, junto a los usos cada vez más complejos que realizan los consumidores de los diversos medios que tienen a su alcance, han sido señaladas como algunas de las causas principales por las que las organizaciones están reorientando sus presupuestos destinados a comunicaciones de marketing hacia los denominados medios alternativos (entre los que se incluye el emplazamiento de producto, el marketing de eventos o el marketing móvil). Esta tendencia queda bien reflejada en el crecimiento de la inversión en medios alternativos durante los últimos años a nivel mundial, que ha alcanzado un promedio del 21,7\% desde 2002 hasta 2007 (PQ Media, 2008). En este sentido, una marca de referencia como Coca-Cola anunció ya hace años que iba a incrementar sus recursos publicitarios en videojuegos y DVDs (Grover et al., 2004).

Dentro de estos medios alternativos, el desarrollo de videojuegos publicitarios (advergames) está adquiriendo una relevancia especial en los últimos años. De hecho, la inversión en advergames figura en la tercera posición dentro de las herramientas que se espera que más crezcan en los próximos cinco años (PQ Media, 2008). Varios factores pueden explicar este hecho. Primero, existen cada vez más videojugadores de todas las edades, y en determinados segmentos demográficos incluso se está produciendo una migración de los medios tradicionales (como la televisión) a estos formatos interactivos, por lo que las comunicaciones de marketing mediante advergames pueden alcanzar a consumidores que están cambiando sus hábitos de consumo mediático (Díaz-Nosty, 2006; Selva, 2009). Segundo, la multiplicidad de plataformas en la que estos advergames pueden ser consumidos (ordenador personal, videoconsolas, Web, televisión interactiva o teléfono móvil, entre otros) permite alcanzar virtualmente a cualquier consumidor. Finalmente, hay que reseñar su versatilidad en cuanto a géneros (sirvan como ejemplo los videojuegos de lucha, deportivos, aventuras gráficas o puzzles), lo que permite personalizar la comunicación en función de las preferencias de múltiples usuarios.

Este interés de los anunciantes por los advergames queda reflejado en el hecho de que las principales agencias de medios y de publicidad hayan abierto en los últimos años divisiones especializadas en advergaming o en emplazamiento publicitario en videojuegos (por ejemplo, The Bounce Interactive Gaming Group (BIG), perteneciente a Young \& Rubicam, o Play Division de Starcom Media). Sin embargo, este interés empresarial no se ha traducido en una mayor atención desde el punto de vista académico, donde apenas existen trabajos que hayan tratado de abordar conceptualmente el tema. 
Así, el presente artículo tiene como objetivo general realizar una delimitación conceptual de los advergames como herramienta de las comunicaciones de marketing. Para ello, la estructura del trabajo será la siguiente. En primer lugar, se define la herramienta y se describen las diferentes tipologías de advergames existentes. A continuación, se revisan los efectos que la utilización de advergames puede ejercer sobre diferentes objetivos de marketing y de comunicación. Finalmente, en el apartado de conclusiones, se apuntan algunas recomendaciones a la hora de desarrollar este tipo de comunicaciones de marketing, así como las cuestiones éticas y morales que deben considerarse.

\section{CONCEPTUALIZACIÓN DEL ADVERGAME}

\subsection{Definición y características principales}

La práctica de desarrollar videojuegos para promocionar productos o servicios es tan antigua como el propio nacimiento de los videojuegos. Así, Grundy (2008) afirma que el primer advergame de la historia fue probablemente Datsun 280 Zzzap, un videojuego lanzado en 1976 para promocionar el Datsun $280 \mathrm{Z}$. Otros de los primeros advergames conocidos fueron Tooth Protectors (creado en 1982 por Johnson \& Johnson); Chase the Chuckwagon (creado también en 1982 por Ralston-Purina); y el conocido Pepsi Invaders (desarrollado en 1983 por Atari para Coca-Cola, que fue regalado a los asistentes a una convención de ventas en Atlanta). Estudios recientes han conceptualizado el advergaming (Selva, 2009; Méndiz, 2010) en el marco de la convergencia actual entre contenido publicitario y contenido editorial, mostrando sus relaciones con otros formatos como el advertainment (Martí, 2002; Russell, 2007; Martorell, 2009; Pineda y Ramos, 2009) y situándolo así en la órbita de lo que se ha denominado branded content (Russell, 2007; Martí y Muñoz, 2008).

El branded content, denominado por la industria branded entertainment, ha sido definido, como "una estrategia de marketing que mezcla publicidad y entretenimiento para alcanzar y comprometer a los consumidores, construir notoriedad de marca y crear asociaciones de marca positivas que puedan incrementar las ventas en los consumidores" (PQ Media, 2010:9). Se trata de una estrategia que se desarrolla pues en el contexto de una creciente convergencia entre los medios de comunicación y los productores de contenido (Donaton, 2004) y cuyo impacto en los medios de comunicación ha provocado que en los últimos años se esté hablando ya de brandcasting (frente al concepto clásico de broadcasting) como de un modelo en el que el contenido está "controlado y, normalmente, (es) propiedad del anunciante" (Greenwood, 2006). Unos contenidos para los que se ha estimado un crecimiento de la inversión, a nivel mundial, en los próximos años de en torno al 9.1\% hasta el 2014 (PQ Media, 2010) y en los que el advergaming ocupa un lugar destacado.

El término advergame es un neologismo inglés acrónimo de advertisement (anuncio) y videogame (videojuego), ofreciéndose una muestra de las principales definiciones propuestas en la literatura en el cuadro 1. 
Tabla 1

Definición de advergame

\begin{tabular}{|c|c|}
\hline Investigadores & Definición \\
\hline Elkin (2002) & $\begin{array}{l}\text { "Juegos específicamente diseñados como parte de la campaña } \\
\text { en el medio online de un anunciante" }\end{array}$ \\
\hline Hernández et al. (2004) & $\begin{array}{l}\text { "La distribución de mensajes publicitarios a través de juegos } \\
\text { electrónicos" }\end{array}$ \\
\hline Dahl et al. (2006) & $\begin{array}{l}\text { "Una forma de comunicación de marketing que comprende } \\
\text { mensajes comerciales embebidos en el contenido de videojue- } \\
\text { gos a la venta y juegos electrónicos online" }\end{array}$ \\
\hline Moore (2006) & "Videojuegos patrocinados por anunciantes" \\
\hline Winkler y Bucker (2006) & $\begin{array}{l}\text { "Juegos online diseñados con propósitos específicos de marke- } \\
\text { ting de una marca o producto" }\end{array}$ \\
\hline Mallinckrodt y Mizerski (2007) & $\begin{array}{l}\text { "Una forma de entretenimiento de marca que presenta mensajes } \\
\text { publicitarios, logos y mascotas en un formato de juego" }\end{array}$ \\
\hline Wise et al. (2008) & "Un videojuego desarrollado en torno a una marca" \\
\hline Selva (2009) & $\begin{array}{l}\text { "Un videojuego financiado por un anunciante y creado para un } \\
\text { producto o marca con un determinado objetivo publicitario" }\end{array}$ \\
\hline
\end{tabular}

Fuente: elaboración propia

Como puede observarse, en la literatura no siempre se ha establecido claramente la diferenciación entre advergame y emplazamiento de producto en videojuegos, pese a tratarse de conceptos distintos. Así, aunque ambos se caracterizan por la utilización de videojuegos como medio de difusión de comunicaciones de marketing o mensajes publicitarios, en el caso del advergaming estos videojuegos son desarrollados ex profeso para difundir los mensajes, mientras que en el emplazamiento de producto en videojuegos el videojuego se desarrolla independientemente de la acción de comunicación de las marcas que, posteriormente, son emplazadas en él.

Estas dos características principales que definen a los advergames (el soporte de difusión del mensaje y la intencionalidad del desarrollo del videojuego) hace que las comunicaciones de marketing mediante advergames se desarrollen en el marco de los denominados mensajes híbridos, definidos como "todo intento pagado de influir a las audiencias para obtener un beneficio comercial usando comunicaciones que presentan un carácter no comercial (Balasubramanian, 1994, p. 29). La naturaleza de estos mensajes híbridos combina las ventajas de dos de las grandes herramientas de comunicación no personal utilizadas tradicionalmente por las organizaciones: la publicidad y la publicity. Por una parte, estos mensajes son pagados por la organización, lo que le permite un control sobre el mismo (publicidad); por otra, la organización no es identificada claramente como emisor del mensaje, por lo que su aparente falta de intención comercial (publicity) puede redundar en una mayor credibilidad hacia el mensaje por parte del receptor (Balasubramanian, 1994).

Otra característica de los advergames que conviene destacar es la aproximación indirecta que proporcionan. Dado que el mensaje no se comunica explícitamente en el anuncio sino que es potenciado por el contexto (en este caso del videojuego), se suelen generar menos res- 
puestas cognitivas desfavorables que mediante una aproximación directa (Yi, 1990). Asimismo, puesto que el foco de atención principal es el contenido de entretenimiento (videojuego), el foco secundario (comunicación de marca) se procesa más implícitamente, pudiendo evitarse en mayor grado procesos como la contrargumentación del consumidor al mensaje. Este hecho está en consonancia con la ruta periférica hacia la persuasión (Petty et al., 1983), ya que se ha detectado que los emplazamientos naturales no despiertan la contrargumentación y es más probable que produzcan efectos afectivos positivos. Estos sentimientos positivos se ven potenciados por el entretenimiento que proporcionan los advergames, ya que se sabe que los contextos que favorecen estados de ánimo positivos repercuten favorablemente en la evaluación del mensaje (Petty et al., 1983). En concreto, se ha sugerido que "si el juego es entretenido y el jugador está pasando un buen rato, los sentimientos positivos hacia el juego deberían extenderse hacia los productos anunciados en el juego" (Glass, 2007, p. 5).

\subsection{Tipología de advergames}

El desarrollo de un advergame implica la planificación del desarrollo de un videojuego en el que se pueda integrar de forma óptima la marca o producto del anunciante en función de determinados objetivos de marketing. Ahora bien, esta integración admite ciertas gradaciones hasta el punto de que se ha sugerido que "el hecho de que un advergame sea encargado específicamente para una marca no siempre implica un protagonismo de ésta" (Selva, 2009, p. 155). En este sentido, Chen y Ringel (2001) han señalado tres niveles de integración de la marca o producto en un advergame: a) asociativa, en la que la marca/producto es emplazada para asociarla a la actividad que se desarrolla en el videojuego, b) ilustrativa, en la que la marca/producto desempeña un papel relevante en el videojuego y c) demostrativa, en la que el jugador puede experimentar la marca/producto en su contexto natural, reproducido por el entorno del videojuego. Estos tres niveles de integración marca-videojuego darían lugar a tres tipos de advergames, resumiéndose sus principales características en el cuadro 2. Estas categorías no son mutuamente excluyentes, sino que pueden combinarse entre sí. Las dos primeras requieren un menor desembolso económico, y están más orientadas a la percepción de marca, mientras que la última exige un mayor desembolso económico pero puede conseguir una mayor implicación del videojugador con el producto/marca.

Tabla 2

Tipos de advergames

\begin{tabular}{|c|c|c|}
\hline Tipo de advergame & Características & Ejemplo \\
\hline ASOCIATIVO & $\begin{array}{l}\text { La marca o producto se emplaza en el } \\
\text { contexto del juego. }\end{array}$ & $\begin{array}{l}\text { Emplazamiento de vallas publicita- } \\
\text { rias de una marca en los escenarios } \\
\text { del videojuego. }\end{array}$ \\
\hline ILUSTRATIVO & $\begin{array}{l}\text { La marca o producto emplazado des- } \\
\text { empeña un papel relevante en el juego. }\end{array}$ & $\begin{array}{l}\text { Emplazamiento del logo de una mar- } \\
\text { ca (o del propio producto) que aporta } \\
\text { puntos al videojugador. }\end{array}$ \\
\hline DEMOSTRATIVO & $\begin{array}{l}\text { El jugador experimenta el producto o } \\
\text { marca en el contexto del videojuego. }\end{array}$ & $\begin{array}{l}\text { Emplazamiento de un modelo de } \\
\text { coche que el videojugador puede } \\
\text { conducir durante el juego. }\end{array}$ \\
\hline
\end{tabular}

Fuente: Basado en Chen y Ringel (2001) 
Los advergames asociativos se corresponden con el emplazamiento de producto tradicional en otros medios como el cine o la televisión. En un advergame de estas características el anunciante desarrolla un videojuego que le permita emplazar su marca o producto de un modo secundario, por ejemplo mediante vallas publicitarias, carteles o cualquier otro formato (generalmente gráfico), en el contexto del juego. Según Chen y Ringel (2001) este tipo de emplazamiento es más efectivo cuando la categoría de producto de la marca emplazada es congruente con el tema del videojuego (por ejemplo, una marca de carburante en un videojuego de carreras).

Los advergames ilustrativos serían el equivalente del llamado emplazamiento integrado en la trama (Russell, 2002). En este caso, la marca o producto está integrada en el desarrollo del advergame y desempeña un papel más o menos relevante en él (por ejemplo, en el advergame Oreo Adventure el jugador suma puntos si consigue recoger diferentes productos de la marca, como galletas o envoltorios, emplazados en el juego).

En tercer lugar, los advergames demostrativos permiten al usuario interactuar con la marca/producto emplazado. Así, el videojugador puede manipular recreaciones virtuales del producto e incluso experimentar simulaciones de consumo (por ejemplo, conducir un modelo virtual de un vehículo en un advergame de carreras de coches como en el $B M W M 3$ Challenge desarrollado por BMW).

\section{APORTACIONES DE LOS ADVERGAMES A LAS COMUNICACIONES DE MARKETING}

Los objetivos de una organización a la hora de utilizar un advergame en sus comunicaciones de marketing pueden ser diversos: llegar de una manera más eficaz a sus públicos de interés, huir de la saturación publicitaria y de los costes de otros medios o construir mensajes que logren una mayor interacción e implicación de los consumidores, entre otros (Martí, 2010). En el cuadro 3 se recogen los principales objetivos de marketing y de comunicación que pueden alcanzarse mediante el uso de advergames, así como la contribución de éstos a la consecución de dichos objetivos.

Tabla 3

Principales objetivos que se pueden alcanzar mediante advergames

\begin{tabular}{ll}
\hline \multicolumn{1}{c}{ Objetivos } & \multicolumn{1}{c}{ Contribución de los advergames } \\
\hline $\begin{array}{l}\text { Evitar la saturación } \\
\text { publicitaria }\end{array}$ & $\begin{array}{l}\text { En los advergames el contenido publicitario está integrado en el } \\
\text { contenido de entretenimiento. } \\
\text { Internet se consolida como un medio de comunicación importan- } \\
\text { Orientación al ecosistema } \\
\text { mediático actual }\end{array}$ \\
$\begin{array}{l}\text { te y los videojuegos online ganan popularidad. Los advergames } \\
\text { se adaptan a este contexto mediático. }\end{array}$ \\
$\begin{array}{l}\text { Los advergames implican un coste mucho menor que el de otras } \\
\text { herramientas como los spots televisivos. }\end{array}$ \\
$\begin{array}{l}\text { Alcance de nuevos públicos costes } \\
\text { objetivo }\end{array}$ & $\begin{array}{l}\text { Cada vez más segmentos de mercado son consumidores de } \\
\text { videojuegos. }\end{array}$ \\
\hline
\end{tabular}




\begin{tabular}{ll}
\hline \multicolumn{1}{c}{ Objetivos } & \multicolumn{1}{c}{ Contribución de los advergames } \\
\hline $\begin{array}{l}\text { Mayor exposición al } \\
\text { consumidor }\end{array}$ & $\begin{array}{l}\text { Los advergames permiten mayores períodos de exposición a la } \\
\text { marca/producto que otras herramientas. }\end{array}$ \\
$\begin{array}{l}\text { Interacción con la marca/ } \\
\text { producto }\end{array}$ & $\begin{array}{l}\text { Los advergames permiten interactuar con la marca/producto, lo } \\
\text { que tiene efectos positivos sobre la memoria y las actitudes. } \\
\text { Mejorar el control de la } \\
\text { eficacia publicitaria }\end{array}$ \\
$\begin{array}{l}\text { Los advergames online permiten controlar la eficacia publicitaria } \\
\text { en tiempo real. }\end{array}$ \\
Lotoriedad de marca \\
$\begin{array}{l}\text { Los advergames pueden contribuir a incrementar la notoriedad } \\
\text { de marca gracias a la interactividad, el nivel de información }\end{array}$ \\
sobre la marca, la integración con la trama y un mayor tiempo de \\
exposición. \\
Recuerdo/reconocimiento de \\
Los advergames tienen efectos positivos sobre la memoria, po- \\
tenciados al incluirse sólo una marca en el videojuego.
\end{tabular}

Fuente: elaboración propia

\section{Evitar la saturación publicitaria}

Según datos de Initiative Media, el telespectador español medio está expuesto a 92 anuncios de televisión al día (642 a la semana, 33.384 al año), lo que supone más de 30 minutos de su consumo televisivo diario. El problema principal para las organizaciones radica no sólo en que muchos de sus esfuerzos en comunicaciones de marketing quedan diluidos entre el resto de mensajes, sino que en muchas ocasiones éstos son deliberadamente ignorados por unos consumidores hastiados por la saturación publicitaria. Conocidos comportamientos del consumidor en este sentido son el zapping (cambio de canal cuando comienzan las pausas publicitarias) o el zipping (avance rápido de la programación televisiva grabada por el telespectador). En este sentido, los advergames (como mensajes híbridos) evitan este tipo de comportamientos del consumidor puesto que el contenido publicitario está integrado en el contenido de entretenimiento.

\section{Orientación al ecosistema mediático actual}

El consumo de medios ha cambiado sustancialmente en los últimos años, en especial debido a la consolidación de medios como Internet, que empieza a ser un medio importante en el share multimedia diario situándose incluso por encima de los medios impresos (Díaz- 
Nosty, 2006). Dentro de los contenidos más buscados en la red se hallan los videojuegos. En este contexto, las comunicaciones de marketing a través de advergames online pueden alcanzar a consumidores que dedican cada vez más tiempo a jugar a videojuegos en Internet y menos a consumir otros medios. Este potencial de los advergames como herramienta de comunicación se justifica también por la popularidad que están adquiriendo los videojuegos entre la población española. Así, en España según GFK-Emer (cfr. en UEM, 2006) son ya 8,8 millones los usuarios habituales de los videojuegos (un 20\% de la población), de los que casi la mitad (42\%) son mayores de 18 años. Es decir, en el complejo ecosistema mediático actual, los videojuegos van ganando atención por parte de cada vez más segmentos de consumidores y una orientación al mercado pasa ineludiblemente por tener en consideración en el plan de comunicaciones de marketing un "espacio" en el que cada vez hay más consumidores. Además, la gratuidad de los advergames permite a los anunciantes llegar a todo el mundo (frente a otras estrategias de publicidad en videojuegos como el emplazamiento de producto en títulos comerciales, que sólo llegan a los videojugadores que compran el videojuego).

\section{Reducción de costes}

La reducción de costes es un objetivo que habitualmente persiguen las organizaciones de cara a mejorar su competitividad. En cualquier ciclo económico, y en especial en los períodos de crisis, esta reducción suele afectar principalmente al departamento de marketing y, en concreto, a las acciones de comunicación y publicidad (Fill, 2006). En este sentido, la utilización de los videojuegos como herramienta de las comunicaciones de marketing puede aportar soluciones a este problema debido a los ventajosos costes que presenta frente a otras herramientas como, por ejemplo, los spots televisivos. A título ilustrativo, el desarrollo de un advergame en España se sitúa en una horquilla que va desde los 2.000 a los 40.000 euros. Estos costes parecen ofrecer un buen retorno de la inversión, lo que sitúa a los advergames en clara ventaja frente a otras herramientas como los spots televisivos, los anuncios en prensa e incluso el propio emplazamiento de producto en videojuegos para videoconsolas (IGDA, 2005).

\section{Alcance de nuevos públicos objetivo}

Si en sus inicios el consumidor mayoritario de videojuegos era varón de entre 18-35 años, desde hace unos años se puede afirmar que los videojuegos son consumidos virtualmente por cualquier segmento de edad de ambos sexos (Fattah y Paul, 2002). Incluso públicos que tradicionalmente se han mantenido alejados de los videojuegos (especialmente adultos y tercera edad) se están empezando a incorporar como consumidores debido tanto al envejecimiento de la población que empezó a jugar a los videojuegos en su niñez y adolescencia, como a los nuevos públicos que han sabido captar los videojuegos online.

\section{Mayor exposición al consumidor}

Otro posible objetivo de los advergames es conseguir largos períodos de exposición de una marca o producto al consumidor (o consumidor potencial). Este es un objetivo deseado por los anunciantes y es habitual en las campañas publicitarias, puesto que los 
sentimientos de familiaridad derivados de esta exposición repetida pueden ser posteriormente procesados como una preferencia por el estímulo (Zajonc, 1980). En este sentido, los advergames ofrecen unos tiempos de exposición de la marca/producto al consumidor como prácticamente ningún otro soporte puede conseguir (Selva, 2009). Se ha detectado que los videojugadores pueden pasar una media de 5-7 minutos jugando a un advergame, un tiempo al menos 10 veces superior al que dedican a ver un anuncio televisivo (Fattah y Paul, 2002). Estos tiempos de exposición a los advergames pueden ser incluso superiores; por ejemplo, el Toyota Adrenaline consiguió que los videojugadores regresaran al sitio Web a jugar a este advergame de carreras de coches 3 y 4 veces al mes, pasando una media de 20 minutos jugando a él (Marriott, 2001).

\section{Interacción con la marca/producto}

La interactividad, junto con la implicación, ha sido destacada como un elemento que diferencia a los advergames de otras formas de publicidad más estática en Internet como los banners y las pop-ups (Dahl et al., 2006). La interacción con objetos y productos no sólo tiene efectos sobre la memoria sino también sobre las actitudes. En el contexto de los videojuegos, se ha sugerido que la naturaleza interactiva del videojuego consigue que los participantes se impliquen más con los productos, propiciando que los sentimientos positivos inducidos por el videojuego se extiendan hacia los productos emplazados (Homer, 2006). Por ejemplo, mediante el desarrollo de un advergame de carreras de coches, el anunciante puede conseguir que el videojugador interactúe con el próximo modelo que va a lanzar al mercado. De hecho, en el videojuego Lada Racing Club (2006) aparecen coches reales y productos de los proveedores de accesorios en el momento del videojuego en que los videojugadores "tunean" sus propios vehículos; además, el videojuego ofrece precios reales e información de contacto de los proveedores. En el caso de advergames online, éstos pueden incluir enlaces a los concesionarios del área del videojugador o a solicitudes de demostraciones offline.

\section{Mejorar el control de la eficacia publicitaria}

En el caso de advergames online se puede alcanzar un objetivo importante: la medición de la eficacia, en tiempo real, de las comunicaciones de marketing desarrolladas. En las plataformas online (ya sea Web, videoconsola o telefonía móvil), y mediante el registro como usuario como requisito para poder jugar al advergame, no sólo se puede obtener perfiles sociodemográficos y psicográficos del receptor de la comunicación, sino el modo en el que ha interactuado con ella: cuántas sesiones ha jugado; cuánto tiempo ha jugado por sesión; el tiempo medio de juego de las sesiones; cuánto tiempo ha permanecido observando un emplazamiento de marca o producto (por ejemplo una valla publicitaria); con cuáles de nuestros productos ha interactuado, cuánto tiempo y de qué modo, etc.

\section{Notoriedad de marca}

La notoriedad de marca se puede definir como "la intensidad de la marca en la memoria, reflejada por la habilidad para identificar la marca en diferentes situaciones" (Keller, 1993 , p. 3). Ya se ha señalado cómo los advergames pueden aumentar el conocimiento que 
un consumidor tiene sobre las marcas y productos de una organización (por ejemplo, a través de la interacción durante el juego). El nivel de información que se puede ofrecer sobre estas marcas o productos en un advergame es mucho mayor que el que se puede ofrecer a través de otras herramientas como el spot televisivo o un anuncio en radio. Además, si la marca/producto está bien integrado en la trama del videojuego, este conocimiento hacia el mismo se desarrolla de manera natural y con un grado de implicación por parte del videojugador previsiblemente mucho mayor que en otras herramientas de las comunicaciones de marketing. Estos factores, unidos a mayores tiempos de exposición, se traducirán en un mayor grado de notoriedad de marca por parte del videojugador.

\section{Recuerdo/reconocimiento de marca}

Los emplazamientos de marcas y productos en advergames afectan al recuerdo y reconocimiento de estas marcas y productos, puesto que se ha detectado que tienen efectos sobre la memoria de los videojugadores (Nelson, 2002; Winkler y Buckner, 2006; Yang et al., 2006). Aunque los estudios académicos efectuados han mostrado resultados desiguales, todos corroboran la existencia de efectos positivos sobre la memoria. Por ejemplo, Nelson (2002) demostró en su trabajo experimental que los videojugadores recordaban aproximadamente un $30 \%$ de las marcas que aparecían en el videojuego. Winkler y Buckner (2006) también demostraron que el recuerdo de producto/marca en los advergames es mejor que en los videojuegos en general, posiblemente porque en los advergames sólo se suele insertar una marca/producto. Finalmente, Yang et al. (2006) muestran que los advergames provocan un nivel bajo de memoria explícita sobre las marcas emplazadas, aunque influyen en la memoria implícita del videojugador.

\section{Familiaridad con la marca}

Las comunicaciones de marketing mediante advergames también pueden mejorar la familiaridad de los consumidores con las marcas/productos de las organizaciones. La familiaridad ha sido definida como el número de experiencias relacionadas con el producto que ha acumulado un consumidor (Alba y Hutchinson, 1987). El conseguir que los consumidores desarrollen un alto grado de familiaridad hacia una marca o producto es importante puesto que se sabe que los estímulos familiares son posteriormente procesados como una preferencia por dicho estímulo (Zajonc, 1980). Por el momento se carece de pruebas empíricas que determinen en qué grado la exposición e interacción de los videojugadores con las marcas emplazadas en advergames afectan a la familiaridad. No obstante, dadas las características de los videojuegos (elevados tiempos de exposición, repetición del estímulo e interacción) es lógico esperar que los advergames ayuden a desarrollar sentimientos de familiaridad hacia las marcas/productos emplazados en ellos y, por tanto, a procesarlos posteriormente de manera favorable para las organizaciones.

\section{Identificación con la marca}

Uno de los objetivos prioritarios de las organizaciones actuales es conseguir una identificación total consumidor-marca/organización con el fin de ganar una importante ventaja 
competitiva (Bhattacharya y Sen, 2003). Esta ventaja radica en que dicha identificación no sólo se basa en parámetros racionales sino en importantes aspectos psicológicos y emocionales que permiten desarrollar vínculos más fuertes entre las organizaciones y los consumidores (Marín y Ruiz, 2007). Los consumidores identificados con sus marcas son consumidores leales (protegiendo a la marca de la competencia), buscan el bien de la marca porque se consideran parte de ella y no escatiman esfuerzos en captar nuevos consumidores para "sus" marcas (Bhattacharya y Sen, 2003). Una de las teorías en las que se basa este hecho, la teoría de la identidad social, determina que los individuos se sienten atraídos por organizaciones y por otros individuos cuando perciben que tienen rasgos y características similares a los suyos y, por tanto, con los que son capaces de compartir sentimientos, opiniones o valores (Marín y Ruiz, 2007). El contexto narrativo de muchos géneros de advergames puede ayudar a desarrollar esta identificación positiva marca-consumidor; por ejemplo, a través de los personajes o mascotas que protagonizan los videojuegos las organizaciones pueden comunicar identidades de marca, estilos de vida y modos de comportamiento que incentiven esta identificación.

\section{Actitud hacia la marca}

En el contexto de los videojuegos, se ha detectado que los videojugadores pueden desarrollar una actitud positiva hacia las marcas emplazadas en el videojuego al que han jugado. En esencia, un advergame es un videojuego cuya principal misión es divertir al videojugador; en la medida en que el sujeto tenga una actitud positiva hacia el advergame, aumenta la posibilidad de que el individuo transfiera esa actitud positiva hacia la marca protagonista del juego, vía asociaciones simples o aprendizaje asociativo. Por ejemplo, Glass (2007) detectó que los participantes en su estudio categorizaron a las marcas emplazadas como "buenas" más rápidamente que como "malas". El autor controló que esta actitud no estaba basada en sentimientos positivos previos hacia estas marcas, ya que en un cuestionario completado con anterioridad a jugar al videojuego los participantes mostraron actitudes más positivas hacia las marcas que no estaban emplazadas en el videojuego. En otro estudio, Anderson y Bushman (2001) mostraron que la exposición a los videojuegos está positivamente relacionada con la activación psicológica (como componente de la experiencia afectiva), por lo que la exposición repetida a marcas emplazadas podría provocar mayores preferencias hacia la marca. Por último, Mallinckrodt y Mizerski (2007) detectaron que los niños que jugaron al advergame en el que aparecía una marca de cereales mostraron una preferencia altamente significativa a la hora de elegir estos cereales frente a otras marcas de cereales y otros tipos de alimento.

\section{CONCLUSIONES E IMPLICACIONES}

En un entorno como el actual, caracterizado por una demanda que exige experiencias personalizadas que aporten valor añadido al consumidor y habituada al uso de las nuevas tecnologías, la comunicación de masas a través de los medios tradicionales está perdiendo eficacia. Este contexto, sin embargo, es el escenario idóneo para el desarrollo de nuevas fórmulas de comunicación interactivas que se integren en los hábitos cotidianos del consu- 
midor. Una de estas fórmulas es el advergaming, que presenta un extraordinario potencial de desarrollo. Muestra de ello es que los advergames están ganando terreno en los presupuestos de las comunicaciones de marketing de las organizaciones (emarketer, 2009).

No obstante, manejar de manera eficaz esta potente herramienta de las comunicaciones de marketing exige un importante trabajo por parte de las organizaciones ya que desarrollar un advergame no consiste únicamente en desarrollar un videojuego en el que emplazar la marca o producto. En primer lugar, resulta necesario tener bien claro el objetivo (o los objetivos) de marketing de la organización. Estos objetivos pueden ser muy diversos, como alcanzar nuevos públicos objetivo, conseguir una mayor interacción del consumidor con las marcas/productos de la organización, aumentar la notoriedad de la marca, aumentar la identificación con la marca o mejorar la actitud hacia la marca. Los objetivos seleccionados condicionarán los factores de diseño del advergame que, a su vez, pondrán en marcha diversos procesos cognitivos del consumidor con efectos diferentes sobre su memoria y sus actitudes.

Otro factor importante a tener en cuenta a la hora de desarrollar un advergame es conocer las características sociodemográficas y psicográficas del público objetivo al que va dirigida la comunicación. Identificar la tipología de videojugador (videojugadores asiduos o videojugadores espóradicos) o si consume los videojuegos online u offline también resultará determinante a la hora de elegir tanto el género como la modalidad de difusión del advergame. Un tercer factor de importancia es conocer la plataforma (ordenador personal, videoconsola o teléfono móvil, entre otros) a través de la cual consume habitualmente videojuegos el público objetivo al que va dirigida la comunicación.

Por otra parte, los advergames deben ser contemplados desde una perspectiva holística e integrada en el resto de acciones de comunicación de la organización y no como una acción puntual y aislada. Este marco de trabajo holístico también debe permitir un uso sinérgico de todos los medios a disposición tanto de la organización como del consumidor. Por ejemplo, un consumidor sin acceso doméstico a Internet puede acceder al advergame de la organización mientras navega desde la oficina para, desde allí, descargar una versión a su teléfono móvil. O también puede descargarse vía bluetooth este advergame a su teléfono móvil desde una marquesina interactiva. Cuanto más se facilite el trasvase del advergame entre las distintas plataformas más se potenciará su consumo por parte de los usuarios. Además, cada medio posee sus propias características que pueden ser utilizadas para alcanzar distintos tipos de interacciones y objetivos del advergame con el consumidor.

Desde un punto de vista académico, son necesarios nuevos trabajos teóricos que profundicen en la naturaleza de estos videojuegos orientados a las comunicaciones de marketing, así como en sus características y tipología. También son necesarios más trabajos empíricos que arrojen luz sobre aspectos que permanecen todavía parcial o totalmente inexplorados. Los efectos de una exposición continuada y planificada de los advergames sobre la memoria y las actitudes de los consumidores; el papel moderador del género de videojuego sobre los diferentes objetivos de marketing; los efectos de la modalidad del emplazamiento (visual, auditiva o audiovisual) sobre la memoria y las actitudes de los consumidores; o el posible apego parasocial desarrollado entre los videojugadores y los personajes de los advergames (y sus efectos sobre las actitudes) son aspectos sobre los que se requiere un mayor conocimiento si se pretende aumentar la eficacia de este tipo de comunicaciones de marketing. 
Desde un punto de vista regulatorio, los advergames también plantean retos importantes. La dificultad a la hora de abordar el marco legal que regule este tipo de comunicaciones radica principalmente en la propia naturaleza híbrida de los advergames, la cual no hace fácil la separación entre contenido (videojuego) y publicidad. La necesidad de este marco legal se hace más patente cuando este tipo de comunicaciones de marketing pueden ser dirigidas a públicos objetivo especialmente desprotegidos como los niños. De hecho, se sabe que la utilización de comunicaciones de marketing basadas en advergames es parte fundamental de las campañas de marketing online de productos de alimentación dirigidos a los niños (Moore, 2006; Méndiz, 2010). Esta estrecha relación entre advergaming y público infantil debe ser considerada, además de una oportunidad estratégica (Méndiz, 2010), como una dimensión muy sensible del advergaming que debería ser regulada por los poderes públicos.

Y es que, en nuestra opinión, los advergames deben considerarse como una forma de comunicación comercial, por lo que quedarían sujetos a la legislación y restricciones reservadas a este tipo de comunicaciones. En este sentido, Grimes (2008) propone un marco de trabajo para regular el advergaming, fundamentado en cuatro puntos clave: a) el marco regulatorio que se debe establecer en los medios de comunicaciones de masas en relación al advergaming; b) el marco legal que garantice la defensa del consumidor ante este tipo de comunicaciones; c) la autorregulación que debe realizar el propio sector y d) el marco legal de la relación comunicación/videojugador. Por el momento, y ante el vacío legal existente, la autorregulación del sector es la única vía que está siendo utilizada. Por ejemplo, en noviembre de 2006, 10 miembros de la Children's Food and Beverage Advertising Initiative se comprometieron a no utilizar advergames para promocionar sus productos (Grimes, 2008). No obstante, ni la presencia de marcas/productos en videojuegos ni las comunicaciones comerciales a través de videojuegos quedan reguladas por las directrices de los dos códigos de autorregulación más importantes del sector (la clasificación de videojuegos ESBR adoptada por la Entertainment Software Association y el código PEGI paneuropeo).

Otro de los puntos que plantea serias reflexiones éticas es el marco contractual de este tipo de comunicaciones online. Tanto en los sitios Web como en los advergames online las relaciones entre la organización (por ejemplo un anunciante) y el consumidor se ajustan al marco del contrato de los Términos de Servicio que el usuario acepta a la hora de utilizar dicho servicio (al igual que lo hace al utilizar una cuenta de correo electrónico facilitada por Gmail o Hotmail). Al respecto. Grimes (2008, p. 175) señala que "los niños son raramente alentados a leer los contratos de términos del servicio que, en todo caso, suelen ser inaccesibles para ellos tanto en términos del lenguaje como de la terminología utilizada". Se puede añadir que tampoco los adultos ponen demasiado interés en conocer unos términos de servicio que pueden implicar la aceptación de su monitorización online con fines comerciales.

Nos encontramos pues ante la punta del iceberg de una poderosa herramienta de las comunicaciones de marketing que no sólo plantea interesantes retos, tanto académicos como profesionales, a la hora de desarrollar un marco de trabajo que garantice la máxima eficacia de sus acciones y un ventajoso retorno de la inversión, sino que también va a plantear en los próximos años importantes cuestiones legales y debates éticos y morales en lo referente a su práctica. 


\section{BIBLIOGRAFÍA}

ALBA, J.W. Y HUTCHINSON, J.W. (1987): "Dimensions of Consumer Expertise”, Journal of Consumer Research, Vol. 13, No. 4, pp. 411-454.

ANDERSON, C.A. Y BUSHMAN, B.J. (2001): "Effects of Violent Video Games on Aggressive Behavior, Aggressive Cognition, Aggressive Affect, Physiological Arousal, and Prosocial Behavior: A Meta-analytic Review of the Scientific Literature", Psychological Science, Vol. 12, No. 5, pp. 353-359.

BALASUBRAMANIAN, S.K. (1994): "Beyond Advertising and Publicity: Hybrid Messages and Public Policy Issues", Journal of Advertising, Vol. 23, No. 4, pp. 29-46.

BHATTACHARYA, C. Y SEN, S. (2003): "Consumer-Company Identification: A Framework for Understaning Consumers' Relationships with Companies”, Journal of Marketing, Vol. 67, No. 2, pp.76-88.

CHEN, J. Y RINGEL, M. (2001): “Can Advergaming be the Future of Interactive Advertising?", http://www.locz.com.br/loczgames/advergames.pdf.

DAHL, S.; EAGLE, L. Y BÁEZ, C. (2006): “Analysing Advergames: Active Diversions or Actually Deception", Middlesex University Business School, http://ssrn.com/abstract=907841.

DÍAZ-NOSTY, B. (2006): "Repensar la Comunicación: la Huella es el Mensaje", en Tendencias '06 - Medios de Comunicación, Fundación Telefónica, pp. 15-43.

DONATON, S. (2004): Madison \& Vine: why the Entertainment and Advertising Industries Must Converge to Survive. McGraw-Hill, New York.

ELKIN, T. (2002): “Online Usage Patterns Hit New Milestone in January”, http://www.adage.com/ news.cms?newsId $=34157$.

EMARKETER (2009): In-Game Ads Seek "Next Level” http://www.adweek.com/aw/content_display/news/nontraditional/e3iccea11f70440c0.

FATTAH, H. Y PAUL, P. (2002): "Gaming Gets Serious," American Demographics, Vol. 24, No. 5, pp. 38-44.

FILL, C. (2006): Marketing Communications, Prentice-Hall, Londres.

GLASS, Z. (2007): “The Effectiveness of Product Placement in Video Games", Journal of Interactive Advertising, Vol. 8, No. 1, http://jiad.org/article96.

GREENWOOD, J. (2006): “Communication 2.0”, Contagious Magazine, pp. 40-45.

GRIMES, S.M. (2008): “Kids' Ad Play: Regulating Children's Advergames in the Converging Media Context, International Journal of Communications Law and Policy, Vol. 12, pp. 162-178.

GROVER, R.; LOWRY, T.; KHERMOUCH, G.; EDWARDS, C. Y FOUST, D. (2004): “Can Mad Ave. Make Zap-Proof Ads? It's Blurring The Lines Between Promotion and Programming As DVRs Gain Ground”, Business Week, núm. 3868, pp. 36-37.

GRUNDY, J. (2009): “Art History”, en IGDA (2008).

HERNÁNDEZ, M.D.; CHAPA, S.; MINOR, M.S.; MALDONADO, C. Y BARRANZUELA, F. (2004): "Hispanic Attitudes toward Advergames: A Proposed Model of their Antecedents", Journal of Interactive Advertising, Vol. 5, No. 1, http://jiad.org/vol5/nol/hernandez/index.htm.

HOMER, P.M. (2006): "Relationships Among Ad-Induced Affect, Beliefs, and Attitudes: Another Look", Journal of Advertising, Vol. 35, No. 1, pp. 35-51.

IGDA (INTERNATIONAL GAMES DEVELOPERS ASSOCIATION), (2005): Casual Games White Paper, http://www.igda.org/casual/IGDA_CasualGames_Whitepaper_2005.pdf.

IGDA (INTERNATIONAL GAMES DEVELOPERS ASSOCIATION) (2008): Casual Games White Paper, http://www.igda.org/casual/IGDA_CasualGames_Whitepaper_2008.pdf.

KELLER, K. (1993): "Conceptualizing, Measuring and Managing Customer Based Brand Equity", Journal of Marketing, Vol. 57, pp. 1-22. 
MALLINCKRODT, V. Y MIZERSKI, D. (2007): “The Effects of Playing an Advergame on Young Children's Perceptions, Preferences, and Requests", Journal of Advertising, Vol. 36, No. 2, pp. 87-100.

MARÍN, L. Y RUIZ, S. (2007): "La identificación del consumidor con la empresa: más allá del marketing de relaciones", Universia Business Review, No. 13, pp. 62-75.

MARRIOTT, M. (2001): “Untangling the Online Gaming Web," The New York Times, 5 de Julio, D1 (N) G1 (L).

MARTÍ, J. (2002): Publicidad y entretenimiento en la web: Estrategias, Formatos y Tipologías de Advertainment, Tesis Doctoral, Universidad Politécnica de Valencia.

MARTÍ, J. (2010): Marketing y videojuegos: product placement, in-game advertising y advergaming, ESIC.

MARTÍ, J. Y MUÑOZ, P. (2008): Engagement Marketing, Pearson.

MARTORELL, C. (2009): "Y ahora pasamos a publicidad...si usted quiere. El advertainment como alternativa al modelo de comunicación basado en la interrupción". Comunicación presentada en el Congreso Internacional Brandtrends. Universidad Cardenal Herrera - CEU, Valencia, Febrero 2009. http://www.uch.ceu.es/principal/congresos/brandtrends/documentos/comunicaciones_textos/cristina_martorell.pdf

MÉNDIZ, A. (2010): “Advergaming: Concepto, Tipología, Estrategias y Evolución histórica”, Revista Icono 14 , No. 15 , pp. 37-58.

MOORE, E.S. (2006): "It's Child Play: Advergaming and the Online Marketing of Food to Children", The Henry J. Kaiser Family Foundation, http://www.kff.org/entmedia/upload/It-s-Childs-Play-Advergaming-and-the-Online-Marketing-of-Food-to-Children-Bios.pdf.

NELSON, M.R. (2002): "Recall of Brand Placements in Computer/Video Games", Journal of Advertising Research, Vol. 42, No. 2, pp. 80-92.

PETTY, R.E.; CACIOPPO, J.T. Y SCHUMANN, D. (1983): "Central and Peripheral Routes to Advertising Effectiveness: The Moderating Role of Involvement", Journal of Consumer Research, Vol. 10, No. 2, pp. 135-146.

PINEDA, A. Y RAMOS, M. (2009): "El advertainment como técnica de integración de la marca: un estudio de The Call y Mission Zero, de Pirellifilm", Comunicación presentada en el congreso Internacional Brandtrends, Universidad Cardenal Herrera - CEU, Valencia, Febrero 2009. http://www.uch.ceu.es/principal/congresos/brandtrends/documentos/comunicaciones_textos/ antonio_pineda.doc.

PQ MEDIA (2008): “Alternative Media Forecast: 2008-2012”, http://www.pqmedia.com/aboutpress-20080326-amf2008.html.

PQ MEDIA (2010): Global Branded Entertainment Marketing Forecast 2010-2014.

RUSSELL, C.A. (2002): "Investigating the Effectiveness of Product Placements in Televisión Shows: The Role of Modality and Plot Connection Congruence on Brand Memory and Attitude", Journal of Consumer Research, Vol. 29, No. 3, pp. 306-318.

RUSSELL, C. A. (2007): Advertainment: Fusing Advertising and Entertainment, www.bus.umich. edu/.../Advertainment_teaching_materials.pdf.

SELVA, D. (2009): "El videojuego como herramienta de comunicación publicitaria: una aproximación al concepto de advergaming", Comunicación, No. 7, Vol.1, pp. 141-166.

UEM (UNIVERSIDAD EUROPEA DE MADRID), (2006): "Mujeres y Videojuegos: Hábitos y Preferencias de las Videojugadoras", http://www.uem.es/Web/cin/cin2/observatorio/EstudioMujeresyvideojuegos.PDF

WINKLER, T. Y BUCKNER, K. (2006): "Receptiveness of Gamers to Embedded Brand Messages in Advergames: Attitudes towards Product Placement", Journal of Interactive Advertising, Vol. 7, núm. 1, http://www.jiad.org/vol7/nol/winkler/index.htm. 
WISE, K.; BOLLS, P.D.; KIM, H., VENKATARAMAN, A. Y MEYER, R. (2008): “Enjoyment of Advergames and Brand Attitudes: The Impact of Thematic Relevante", Journal of Interactive Advertising, Vol. 9, No. 1, http://www.jiad.org/article107.

YANG, M.; ROSKOS-EWOLDSEN, D.R.;, DINU, L. Y ARPAN, L.M. (2006): “The Effectiveness of 'in-game' advertising: Comparing Collage Students' Explicit and Implicit Memory for Brand Names", Journal of Advertising, Vol. 35, No. 4, pp. 143-152.

YI, Y. (1990): "The Effects of Contextual Priming in Print Advertisements", Journal of Consumer Research, Vol. 17, No. 2, pp. 215-222.

ZAJONC, R.B. (1980): "Feeling and Thinking: Preferences Need no Inferences," American Psychologist, Vol. 35, pp. 151. 